\title{
Copula Function method of the kinematic accuracy of cylindrical roller bearing
}

li lihong ( $\square$ lyh_5204@163.com )

Henan University of Science and Technology https://orcid.org/0000-0001-6961-7277

\section{Yujun XUE}

Henan University of Science and Technology

Jishun LI

Henan University of Science and Technology

\section{Weihang Wang}

Henan University of Science and Technology

\section{Research Article}

Keywords: Copula function, Cylindrical roller bearings, The radial run out of bearings, Joint distribution

Posted Date: March 7th, 2022

DOI: https://doi.org/10.21203/rs.3.rs-1167996/v1

License: (9) This work is licensed under a Creative Commons Attribution 4.0 International License. Read Full License 


\title{
Copula Function method of the kinematic accuracy of cylindrical roller bearing
}

\author{
Lihong LI ${ }^{1} \quad$ Yujun XUE ${ }^{2,3} \quad$ Jishun $^{2 I^{2,3}}$ Weihang WANG ${ }^{1}$ \\ 1.Vehicles \& Transportation Engineering Institute, Henan University of Science and Technology, Luoyang 471003, china \\ 2. Henan Key Laboratory for Machinery Design and Transmission System, Henan University of Science and Technology, Luoyang 471003, China \\ 3. State Key Laboratory of Aviation Precision Bearings, Luoyang LYC Bearings Co., Ltd., Luoyang 471039, China
}

Correspondence to: Lihong Li/ lyh_5204@163.com

\begin{abstract}
The kinematic accuracy of cylindrical rollers bearing isn't only influenced by the size and form precision of its parts, but also more influenced by the cooperation among them. For cylindrical rollers bearing, the main indices of the kinematic accuracy are the ending beat and the radial run out of bearings. There must be dependent relationship between the cooperative action among the parts of bearings and the ending beat or the radial run out of bearings. This relationship is hardly expressed by mathematical formula. However, because the parts dimension deviation and the run out of bearings follow statistic law and have their distribution characteristics, while the copula joint distribution function can connect the run out of bearings with multiple parts dimension accuracy. The copula function is introduced to analyze the dependent relationship between bearing parts cooperative action and its kinematic accuracy. Based on the copula function of statistic theory, the kinematic accuracy of bearings can be forecast by parts geometric accuracy.
\end{abstract}

Keywords: Copula function; Cylindrical roller bearings; The radial run out of bearings; Joint distribution

\section{Introduction}

The kinematic accuracy of bearings isn't only restricted by its parts dimension precision but also influenced by the cooperation among them.

In recent years, many researchers have been studying how to improving the kinematic accuracy from various aspects, example as force, structure,

assembly, lubrication and etc. There were many paper reported about the dependent connection between the parts precision and the bearings

kinematic accuracy [1-15]. But, very few scholars studied the bearing kinematic accuracy from a statistical standpoint. For cylindrical rollers 
bearing, the radial run out and the ending beat are the most important indices influencing on the kinematic accuracy of it. The kinematic accuracy often directly affect machines performance,especially for high-speed and precision instruments [16]. The data from measurement about the radial run out and the ending beat obey statistical distribution laws. Their distribution characters are decided synthetically by bearing parts precision owe to many conditions such as worker technique, environment temperature and humidity, the precision grade of machining tool and so on. On account of various unexpected factors, the part size and form can not be standard value with more or less deviation. While, the bearings is a assembly which consist of multiple parts such as rollers, cage, outer ring, inner ring and etc. When assembling parts together into the bearings, the parts were taken randomly and their precision synthetically decide the bearings kinematic accuracy. So, the kinematic accuracy of the bearings is a comprehensive effect. It needn't only to study the parts precision, but also to study the cooperation among them. While, the relationship between the kinematic accuracy of bearings and the parts precision is quite complex and difficult to be expressed by mathematical model. However, because of their distribution characteristics, the Copula function of joint distribution can connect multiple variables distributions to construct the transmission and map relationship model between the multiple elements precision distribution and the bearing kinematic accuracy. So, the bearing kinematic accuracy can be known with the dimensional precision of parts before assembly.

In 1959, Sklar firstly propounded the copula theory. This theory can be applied to construct Copula function which join or "couple" multivariate distribution functions to their one-dimensional marginal distribution function[17]. In the end of the 20th century, this theory was rapidly developing at home and abroad. Since the 1980s, this theory has been widely applied to insurance, banking business, machine diagnostic system, even to buildings fields, traffic controlling, space technology and so on [18-32]. General distribution algorithm can’t build suitable multivariate JDF (joint distribution function) which can expresses the relationship between it and marginal single distribution functions. But, copula function can construct copula joint distribution function ( CJDF ) which join the multiple dimensional distribution function to the low dimensional marginal function. At the same time, comparing with BOA, PPCA and other algorithms, CJDF can be applied to non parametric estimation of dependence relation between stochastic variables with less operation and can be preferable to give expression to distribution situation of dominant group [33] In this paper, CJDF will be introduced to research distribution characteristics of the inter dependent linkages 
between kinematic accuracy and multiple variables distributions. With this ideas, the kinematic precision of bearings will be approximately

estimated with measuring data about elements dimension deviation before assembly.

\section{Copula Joint Distribution Function}

\subsection{The meaning of CJDF}

As the dependent framework between variables, Copula function includes almost all reliant messages of stochastic variables. When the relationship between variables can't be determined by traditional method, the copula function can be utilized to analyze relevant relationship between variables. CJDF, also called joint distribution function or distribution reliant function, is able to couple the joint distribution function of two or more variables with one dimensional single distribution functions and to distinguish the marginal property from multiple variables distribution. This notion stemmed from Sklar ideas: CJDF is able to be split into one joint function and multiple marginal distribution functions and then, can be applied to analyze dependence among variables [34].

\subsection{Constructing CJDF procedure}

Constructing CJDF need two steps. Firstly, there need one joint distribution function consisted of two or more variables. Secondly, selecting one appropriate copula function by which the joint distribution function of multiple variables is connected to marginal univariant distribution function [35]:

Step one: Joint distribution function

Given random variables xiE $R(i=1,2, \ldots, n)$ with continuous marginal univariant distribution function Yi(xi), which n-dimensional joint distribution function can be set as $\mathrm{G}(\mathrm{x} 1, \mathrm{x} 2, \ldots, \mathrm{xn}),(\mathrm{x} 1, \mathrm{x} 2, \ldots, \mathrm{xn}) \in \mathrm{R}^{\mathrm{n}}$. These exists one unique Copula function written as followed[36]:

$$
C\left(Y_{1}\left(x_{1}\right), Y_{2}\left(x_{2}\right), \ldots, Y_{n}\left(x_{n}\right)\right)=G\left(x_{1}, x_{2}, \ldots, x_{n}\right)
$$

Set $U_{i}=Y_{i}\left(x_{i}\right)(i=1,2, \mathrm{~K}, n)$ then, $x_{i}=Y_{i}^{-1}\left(U_{i}\right)(i=1,2, \mathrm{~K}, n) . \mathrm{Y}^{-1}(\cdot)$ is the inverse function of $\mathrm{Y}(\cdot)$. The equation (1) can be written as: 


$$
\begin{aligned}
& C\left(U_{1}, U_{2}, \ldots, U_{n}\right) \\
= & G\left(Y_{1}^{-1}\left(U_{1}\right), Y_{2}^{-1}\left(U_{2}\right), \ldots, Y_{n}^{-1}\left(U_{n}\right)\right)
\end{aligned}
$$

Step two: The Type selection of Copula function

From the meaning of CJDF, a lot of functions are capable of being used as Copula function, of which there were two kinds functions most researched: $\mathrm{ACF}($ Archimedean Copula function) and $\mathrm{ECF}$ (elliptic Copula function)[36]. The most distinct property of the ECP is that its variable has the same distribution type, moreover, the most common type of the ECP is multiple variables Gaussian Copula (MVG) and multiple variables student's Copula (MVT)[36].

According to the equation (2), multivariate Gaussian Copula function can be written as followed:

$$
\begin{aligned}
& C\left(y_{1}, y_{2}, \ldots, y_{N} ; \rho\right) \\
& \quad=\Phi_{\rho}\left(\Phi^{-1}\left(y_{1}\right), \Phi^{-1}\left(y_{2}\right), \ldots, \Phi^{-1}\left(y_{N}\right)\right)
\end{aligned}
$$

The density function of (3) is written as followed:

$$
\begin{aligned}
& c\left(y_{1}, y_{2}, \ldots, y_{N} ; \rho\right) \\
& =|\rho|^{1 / 2} \exp \left(1 / 2 \tau^{\prime}\left(\rho^{-1}-I\right) \tau\right)
\end{aligned}
$$

Where : $\tau=\left(\tau_{1}, \tau_{2}, \mathrm{~K}, \tau_{N}\right), \tau_{n}=\Phi^{-1}\left(y_{n}\right) \quad(n=1,2, \mathrm{~K}, N)$

$\rho$ is symmetrical positive matrix and every element is 1 in its diagonal line, $|\rho|$ is determinant value of $\rho$. $\Phi_{\rho}($.$) expresses multiple$ variables standard normal distribution function, $\Phi^{-1}\left(y_{n}\right)$ is the inverse function of $\Phi\left(y_{n}\right)$, I is unit matrix.

In building JDF of stochastic variables, CJDF have much superiority:

1 ) CJDF can be used to build JDF flexibly without limiting marginal function type [37];

2 ) when constructuring math model with CJDF, marginal distribution function indicates only individual messages of a single variable, while the correlative messages among variables is able to be described by CJDF. Therefore, one dimensional distribution of variable and 
correlation between variables can be researched individually [37].

\section{Influencing factors on the kinematic accuracy of Cylindrical roller bearing}

There are many influencing factors on the kinematic accuracy of cylindrical roller bearing. Besides load and machine precision, the kinematic accuracy of the bearing is mainly decided by elements geometrical deviation (dimension deviation of rollers, dimension and form deviation of inner ring, dimension and form deviation of outer ring), radial clearance and the number of rollers [38]. When cylindrical bearing running, the kinematic accuracy can also be affected by lubrication, working loading, the assembled pattern, surroundings temperature and pretightening force. In these factors, the elements geometrical deviation is the most important influencing factor. The ending beat and radial run out of bearing are the vital indices of the kinematic accuracy of roller bearings. So, there must be a certain relationship between the kinematic accuracy and the dimension deviation in the radial direction and in the axial direction. To ensure the kinematic accuracy of bearings, the radial run out and the ending beat of bearings need to be controlled to a certain range. So, in the paper, the relation between the radial and ending beat of bearings and the dimension deviation of bearing elements will be analyzed to control the kinematic accuracy of bearings.

When the parts has been made, the dimension of them has a certain deviation in precision range because of accidental factors such as temperature, machine accuracy, technique of worker and etc. The deviation value is stochastic and follows the statistic law. Before assembling, the dimension distributions of parts are independent and self-interrelated. While, after being assembled, they influence on each other and interact with each other. This interact would cause bearing radial run out and ending beat and then influence on the kinematic accuracy. The influence on the kinematic accuracy is not single action of one part, but synthetic effect of two or more parts. So, the value of the radial run out and ending beat is also stochastic and follows the statistic law, which must have a relation with the joint distributions of parts. The relationship can be studied by the copula theory.

\section{Data analysis}

\subsection{The stochastic variables}


From the above section, the radial run out and the ending beat of bearing are the main indices influencing on bearing kinematic accuracy.

The influence is a synthetic effect of all bearing parts working. So, without consideration of the influence of other elements, only three elements ( outer ring, inner ring and the rollers) will be taken into account to analyze the kinematic accuracy of bearings. The dimension deviation of them can be measured by precision instrument, so do the the radial run outs and ending beat of bearings. With the statistic theory, the dimension distribution law of them can be gotten by the statistic method, while, with the copula theory and method the the relation between them can be estimated by constructing the mathematics model among them. Then , the kinematic accuracy of the bearings can be known before assembling.

Take the cylindrical roller bearings (the type of bearing is NU1004) for example. Let variable y1 represented the radial run-out and variable y2 represented the ending beat of bearing respectively. Let stochastic variable u represented the upper deviation of inner ring and let stochastic variable $\mathrm{v}$ represented the lower deviation of outer ring respectively. Because every bearing consists of several rollers, the dimension uniformity of rollers is very important factor influencing on the kinematic accuracy of bearings. So, let stochastic variable w represented the absolute value of the difference that is the biggest deviation minus smallest deviation of rollers.

Took 150 groups parts of the bearing to be marked by the number and measured them respectively and gain 150 groups data about $\mathrm{u}$, $\mathrm{v}$, and w. then, these 150 groups parts were assembled together by the No.and 150 bearings were gained. At the same time, the radial run out and the ending beats of bearings were measured in the exclusive instrument[38] and 150 groups data about y1, y2 were also gotten. Only the 10 groups data of $\mathrm{y} 1, \mathrm{y} 2$, and $\mathrm{u}, \mathrm{v}, \mathrm{w}$ were list in table 1 because of the limited space. Even if the measuring instrument is very precise, it has error itself. The 150 groups data had been processed by the error separation method[38]. The following analysis come from processed data.

Table 1. The 10 groups data measured

\begin{tabular}{rrrrrr}
\hline No. & $y_{1}(\mu m)$ & $\mathrm{y}_{2}(\mu m)$ & $u(\mu m)$ & $v(\mu m)$ & \multicolumn{1}{c}{$w(\mu m)$} \\
\hline 1 & 10.8 & 9.6 & 8.61 & 3.81 & 2.91 \\
2 & 9.20 & 3.8 & 4.53 & 5.64 & 3.62 \\
3 & 3.61 & 2.6 & 8.22 & 7.42 & 1.81 \\
4 & 3.82 & 12.3 & 4.61 & 9.11 & 2.84 \\
5 & 5.64 & 5.61 & 3.52 & 10.30 & 7.65 \\
6 & 7.90 & 14.2 & 2.81 & 11.2 & 2.38 \\
7 & 6.81 & 9.04 & 6.90 & 5.63 & 2.82
\end{tabular}




\begin{tabular}{rrrrrr}
8 & 8.52 & 6.51 & 5.81 & 2.80 & 10.55 \\
9 & 2.91 & 1.22 & 3.90 & 4.60 & 8.26 \\
10 & 8.20 & 2.43 & 4.63 & 9.81 & 4.69 \\
\hline
\end{tabular}

\subsection{Data distribution law analysis}

\subsection{1. parameters estimation}

To search for the statistical law of the upper deviation $\mathrm{u}$ of the outer ring diameter, the lower deviation $\mathrm{v}$ of inner ring diameter and the absolute value $\mathrm{w}$ of dimension difference of rollers, the statistic software $\mathrm{R}$ can be utilized to plot histograms of $\mathrm{u}$, v and w. the deviation distribution of $\mathrm{u}, \mathrm{v}$ and $\mathrm{w}$ were displayed in the histograms forms shown in Figure 1. The dotted curves in the Figure 1 indicated the kernel density of $\mathrm{u}, \mathrm{v}$ and $\mathrm{w}$. In the same way, the deviation distribution of $\mathrm{y} 1$ and $\mathrm{y} 2$ were displayed in the histograms forms shown in Figure 2 . The dotted curves in the Figure 2 indicated the kernel density of y1 and y2. From the histogram in figure 1 and figure 2 , the five stochastic variables $\mathrm{u}, \mathrm{v}, \mathrm{w}$ and $\mathrm{y} 1, \mathrm{y} 2$ all approximately complied with normal distribution, but that is only a hypothesis. With the software R, their distributions law had been tested and verified. The results indicated their normal distribution can't be refused as shown in table.2.

In table $2, \mu$ is mean value, $\sigma$ is standard deviation, $\hat{\mu}, \hat{\sigma}$ are estimation of mean value and standard deviation respectively, $\mathrm{P}$ is probability. In normal condition, according to statistics theory, if $\mathrm{P}>0.05$, the above hypothesis is true, or else, $\mathrm{P}<=0.05$, false.

From the table 2, the above hypothesis about normal distribution of $\mathrm{u}, \mathrm{v}, \mathrm{w}$, and $\mathrm{y} 1, \mathrm{y} 2$ can't be refused.
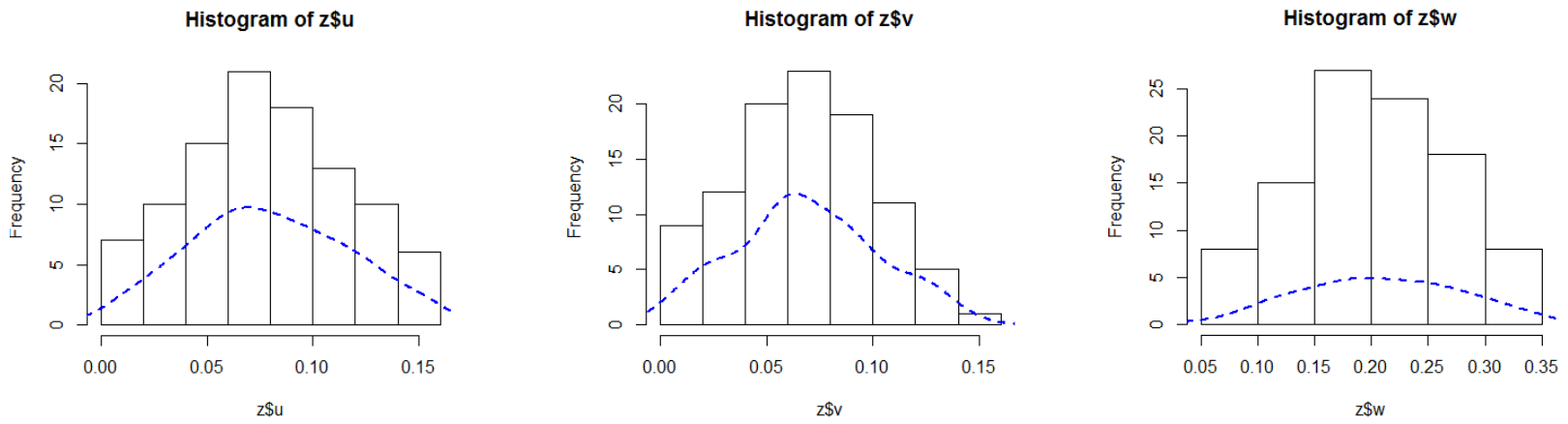

Fig. 1. The distribution and kernel density curves of $\boldsymbol{u}(\mu m), \boldsymbol{v}(\mu m), w(\mu m)$ 
Histogram of $\mathrm{z} \$ \mathrm{y} 1$

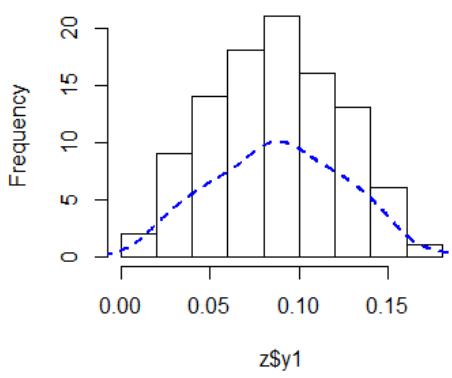

Histogram of $z \$ y 2$

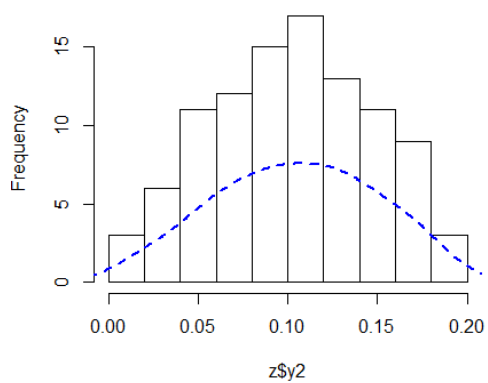

Fig. 2.The distribution and kernel density curves of $y_{1}(\mu \mathrm{m}), y_{2}(\mu \mathrm{m})$

Table 2. Parameters value estimated and verified result

\begin{tabular}{|c|c|c|c|c|c|c|c|}
\hline \multirow[b]{2}{*}{ es } & \multirow[t]{2}{*}{ variabl } & \multicolumn{4}{|c|}{ parameters value estimated } & \multicolumn{2}{|c|}{ verified result } \\
\hline & & $\mu$ & $\sigma$ & $\hat{\mu}$ & $\hat{\sigma}$ & $P$ & $P>0.05$ \\
\hline & $u$ & 7.026 & 2.8236 & 7.0 & 2.637 & 0.2896 & acceptance \\
\hline \multicolumn{8}{|c|}{36} \\
\hline & $v$ & 2.325 & 3.0611 & 2.8 & 3.250 & 0.3592 & acceptance \\
\hline \multicolumn{8}{|c|}{13} \\
\hline & $w$ & 1.543 & 0.4839 & 1.5 & 0.481 & 0.2328 & acceptance \\
\hline \multicolumn{8}{|c|}{46} \\
\hline & $y_{1}$ & 8.274 & 3.9479 & 8.2 & 3.722 & 0.2243 & acceptance \\
\hline \multicolumn{8}{|c|}{86} \\
\hline & $y_{2}$ & 5.395 & 3.0622 & 5.3 & 2.965 & 0.2426 & acceptance \\
\hline \multicolumn{8}{|c|}{94} \\
\hline
\end{tabular}

\subsubsection{Analyzing correlation between stochastic variables}

Considering different extent influence of different variable on the kinematic accuracy, the correlation among the above variables need to be analyzed. With the software R, the covariance matrix between variables $\mathrm{u}, \mathrm{v}$, w and y1, y2 is shown in table 3 . From table 3, y1 has a very strong correlation with $\mathrm{u}, \mathrm{v}, \mathrm{w}$ respectively and similarly, y2 has very strong correlation with $\mathrm{u}, \mathrm{v}, \mathrm{w}$ respectively. Because of the deviation in dimension of bearings parts, the bearings working must cause the radial and axial beat of bearings. The bigger the $\mathrm{u}, \mathrm{v}, \mathrm{w}$, the worse the beat. In addition, from the table 3 , the radial run out has a litter stronger correlation with the upper deviation of outer ring than the lower deviation of inner ring has, similarly, the ending beat has obviously stronger correlation with the upper deviation of outer ring than the lower deviation of inner ring. The 
correlation between the radial run out of bearings and the rollers uniformity is the strongest relation in the first row. The correlation between the rollers uniformity and the ending beat of bearings is also the strongest relation in the second row.

To understand intuitively the correlation between $\mathrm{u}, \mathrm{v}, \mathrm{w}$ and $\mathrm{y} 1, \mathrm{y} 2$. R-Plot can be used to draw the relativity dots plot between them as shown in Figure 3. From Figure 3, y1, y2 have stronger relations to $\mathrm{w}$ than the relation to $\mathrm{u}$ and $\mathrm{v}$ that illustrates the radial run out and the ending beat of bearings bear very stronger correlation to the uniformity of rollers. In the standard deviation range, the smaller w, the more the uniform and the smaller the ending beat.

Table 3. The covariance matrix between variables

\begin{tabular}{llllll}
\hline & $y_{1}$ & $y_{2}$ & $u$ & $v$ & $w$ \\
\hline$y_{1}$ & 1.0000000 & 0.5775901 & 0.4889608 & 0.3035497 & 0.8580857 \\
$y_{2}$ & 0.5775901 & 1.0000000 & 0.4560412 & 0.1583584 & 0.5685766 \\
$u$ & 0.4889608 & 0.4560412 & 1.0000000 & 0.1259457 & 0.4217857 \\
$v$ & 0.3035497 & 0.1583584 & 0.1259457 & 1.0000000 & 0.3650737 \\
$w$ & 0.8580857 & 0.5685766 & 0.4217857 & 0.3650737 & 1.0000000 \\
\hline
\end{tabular}




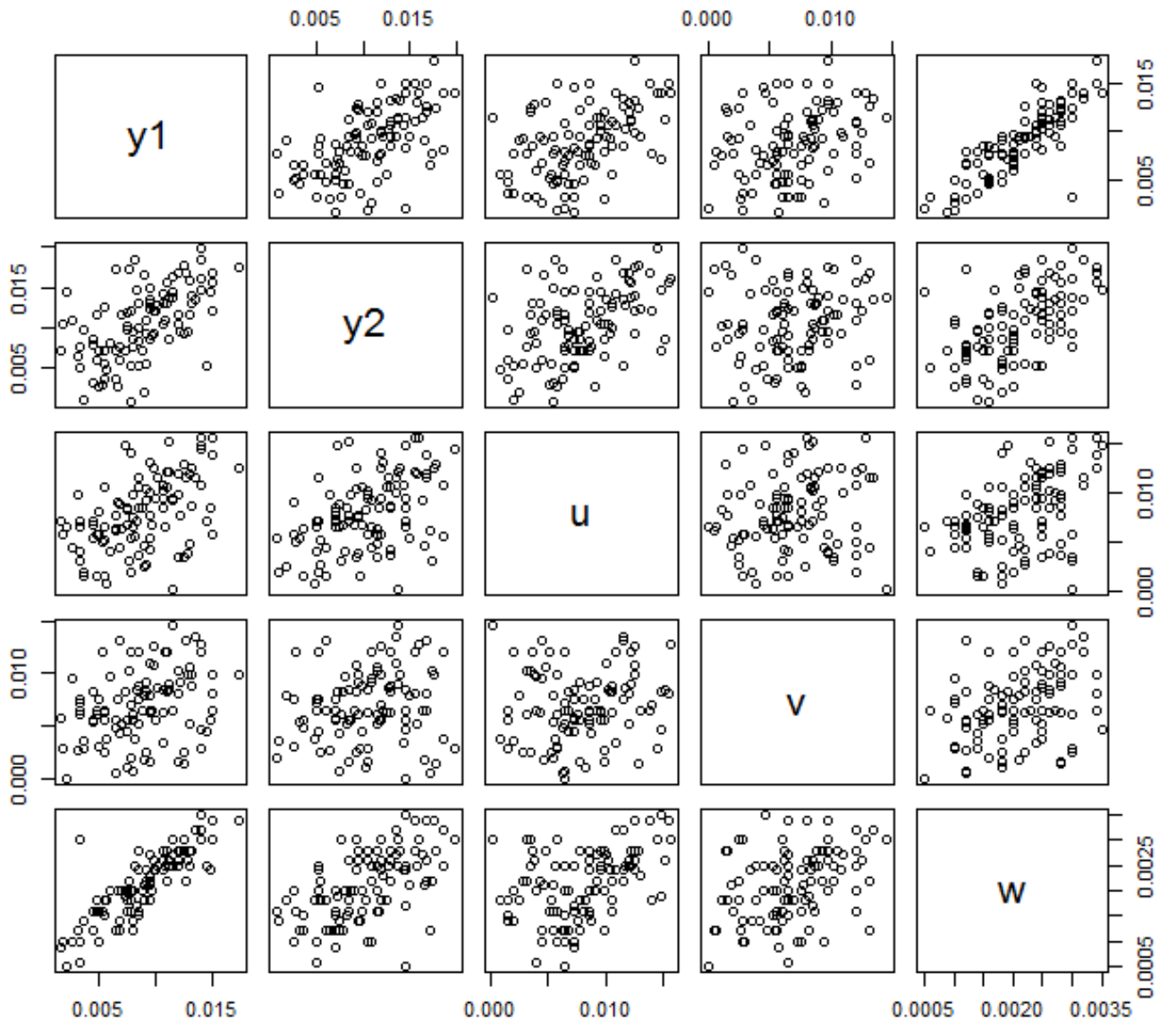

Fig. 3. the relativity between $u, v 、 w$ and $y_{1} 、 y_{2}$ 


\section{Constructing mathematic modeling}

\section{1. variables distribution function}

From the above section, the radial run out of bearings results from the compositive action of bearings parts. The distribution law of the radial run out have connection with the joint distribution of $u, v, w$, then, the distribution function of the radial run out of bearings can be expressed by the joint multivariate distribution function of $u, v, w$. So does the ending beat of bearings. A multivariate distribution function is very difficultly to be described. According to Sklar theory, the CJDF can be utilized to build model to connect the joint distribution function of multiple variables with one-dimensional distribution function. So the distribution function of the radial run out of bearings can be described by the single dimensional marginal distribution function of the upper deviation of outer ring $u$, the lower deviation of inner ring $v$ and the rollers uniformity $w$.

Set the single dimensional marginal distribution functions of the variables $u, v, w$ as $F(u), F(v), F(w)$ respectively. From the section 4.2.1, stochastic variables $u 、 v$, and $w$ approximately follow the normal distribution. According to mathematical statistics, the distribution function of $u 、 v$, and $w$ can be expressed as:

$$
\begin{aligned}
& F(u)=\Phi\left(\frac{u-\mu_{u}}{\sigma_{u}}\right) \\
& F(v)=\Phi\left(\frac{u-\mu_{v}}{\sigma_{v}}\right) \\
& F(w)=\Phi\left(\frac{u-\mu_{w}}{\sigma_{w}}\right)
\end{aligned}
$$

Where $\mu_{u}, \sigma_{u}, \mu_{v}, \sigma_{v}, \mu_{w}, \sigma_{w}$, are the mean values and the standard deviation of $u 、 v 、$ and $w$ respectively, moreover, the density function of $u, v, w$ can be expressed respectively as:

$$
\begin{aligned}
& f(u)=\varphi\left(\frac{u-\mu_{u}}{\sigma_{u}}\right) \\
& f(v)=\varphi\left(\frac{u-\mu_{v}}{\sigma_{v}}\right) \\
& f(w)=\varphi\left(\frac{u-\mu_{w}}{\sigma_{w}}\right)
\end{aligned}
$$

Set the distribution function of the radial run out of bearings as $F\left(y_{1}\right)$ and let $\mathrm{F}(\mathrm{u}, \mathrm{v}, \mathrm{w})$ indicate the joint distribution function of 
variables $\mathrm{u} 、 \mathrm{v}$ and $\mathrm{w}$. From the above stated, there exist the relation between the single dimensional distribution function $F\left(y_{1}\right)$ and multiple dimensional distribution function $F(u, v, w)$. Similarly, set the distribution function of the ending beat of bearings as $F\left(y_{2}\right)$. there also exists the relation between the ending beat distribution function $\quad F\left(y_{2}\right)$ and $F(u, v, w)$. This two relations can be expressed in formula as:

$$
\begin{aligned}
& F\left(y_{1}\right) \sim F(u, v, w) \\
& F\left(y_{2}\right) \sim F(u, v, w)
\end{aligned}
$$

The high dimensional distribution function $F(u, v, w)$ is very complicated to be solved. In general, many mathematics software such as MATLAB and R software only provides two-dimensional copula functions. To be convenient for analysis, the three dimensional joint distribution function $F(u, v, w)$ can be simplified into three items, each of item is one pair-wise joint distribution function containing two-dimension stochastic variables and multiplied by a parameter $\lambda_{i}, \kappa_{i}(\mathrm{i}=1,2,3)$ [39]. Three items of $F(u, v, w)$ can be written as three two-dimensional distribution function $F(u, v), F(v, w), F(w, u)$. Constructing the math modeling is followed as :

$$
\begin{aligned}
& F\left(y_{1}\right)=\lambda_{1} F(u, v)+\lambda_{2} F(v, w)+\lambda_{3} F(w, u) \\
& F\left(y_{2}\right)=\kappa_{1} F(u, v)+\kappa_{2} F(v, w)+\kappa_{3} F(w, u)
\end{aligned}
$$

Where, $\lambda_{i}, \kappa_{i} \quad(\mathrm{i}=1,2,3)$ are revising parameters, and moreover, the density function modeling of $(8)$ are expressed as followed:

$$
\begin{aligned}
& f\left(y_{1}, \mu_{1}, \sigma_{1}\right)=\lambda_{1} f\left(u, v, \rho_{u v}\right) \\
& +\lambda_{2} f\left(v, w, \rho_{v w}\right)+\lambda_{2} f\left(w, u, \rho_{w u}\right) \\
& f\left(y_{2}, \mu_{2}, \sigma_{2}\right)=\kappa_{1} f\left(u, v, \rho_{u v}\right) \\
& +\kappa_{2} f\left(v, w, \rho_{v w}\right)+\kappa_{3} f\left(w, u, \rho_{w u}\right)
\end{aligned}
$$

Where $\rho_{u v}$ is the correlation coefficient variables $u$ and $v, \rho_{v w}$ is the correlation coefficient variables $v$ and $w, \rho_{w u}$ is the correlation coefficient variables $w$ and $u$.

\subsection{Constructing copula function}

From the above section, the CJDF of three dimension can be solved by dimension reduction. The pair-copula function can use a very flexible and intuitive way to construct high dimensional copula function [40]. the pair-wise joint distribution function $F(u, v)$ can be easily connected to its one-dimensional marginal distribution functions $F(u), F(v)$ [41]. There exists only one copula function $C(F(u), F(v))$ set as: 


$$
F(u, v)=C(F(u), F(v))
$$

Similarly, the pair-wise joint distribution function $F(v, w)$ can be connected to its one-dimensional marginal distribution functions $F(v)$ and $F(w)$. The pair-wise joint distribution function $F(w, u)$ can be connected to its one-dimensional marginal distribution functions that are $F(w), F(u)$. There exist copula functions $C(F(v), F(w))$ and $C(F(w), F(u))$ respectively set as:

$$
\begin{aligned}
& F(v, w)=C(F(v), F(w)) \\
& F(w, u)=C(F(w), F(u))
\end{aligned}
$$

From the section 4.2.1, $u 、 v 、 w$ and y1 $、$ y2 satisfied the normal distribution and follow the normal distribution law. From the section 2.2. Gauss copula function can be used to construct mathematical model between the joint distribution function and its marginal distribution function. Now, for Eq. (10) and Eq. (11), each of equation has a Gaussian copula function $C_{G}(F(u), F(v)), C_{G}(F(v), F(w)), C_{G}(F(w), F(u))$, respectively written as:

$$
\begin{gathered}
C(F(u), F(v))=C_{G}(F(u), F(v)) \\
C(F(v), F(w))=C_{G}(F(v), F(w)) \\
C(F(w), F(u))=C_{G}(F(w), F(u)) \\
\left\{\begin{array}{l}
C_{G}(F(u), F(v))=\Phi\left\{F^{-1}(u), F^{-1}(v)\right\} \\
C_{G}(F(v), F(w))=\Phi\left\{F^{-1}(v), F^{-1}(w)\right\} \\
C_{G}(F(w), F(u))=\Phi\left\{F^{-1}(w), F^{-1}(u)\right\}
\end{array}\right.
\end{gathered}
$$

Where $\Phi\{\cdot\}$ is two-dimensional normal distribution function, $\mathrm{F}^{-1}(\mathrm{u})$ is the inverse function of $\mathrm{F}(\mathrm{u}), \mathrm{F}^{-1}(\mathrm{v})$ is the inverse function of $\mathrm{F}(\mathrm{v})$, $\mathrm{F}^{-1}(\mathrm{w})$ is the inverse function of $\mathrm{F}(\mathrm{w})$. The Eq. (13) is expanded as followed: 


$$
\left\{\begin{array}{c}
C_{G}\left(F(u), F(v), \rho_{u v}\right)=\int_{-\infty}^{F^{-1}(u) F^{-1}(v)} \int_{-\infty}^{2 \pi \sigma_{u} \sigma_{v} \sqrt{1-\rho_{u v}^{2}}} \\
\cdot \exp \left\{-\frac{1}{2\left(1-\rho_{u v}^{2}\right)}\left[\frac{\left(r-\mu_{u}\right)^{2}}{\sigma_{u}^{2}}-2 \rho_{u v} \frac{\left(r-\mu_{u}\right)\left(s-\mu_{v}\right)}{2 \sigma_{u} \sigma_{v}}+\frac{\left(s-\mu_{v}\right)^{2}}{\sigma_{v}^{2}}\right]\right\} d r d s \\
C_{G}\left(F(v), F(w), \rho_{v w}\right)=\int_{-\infty}^{F^{-1}(v) F^{-1}(w)} \int_{-\infty}^{2 \pi \sigma_{v} \sigma_{w} \sqrt{1-\rho_{v w}^{2}}} \\
\cdot \exp \left\{-\frac{1}{2\left(1-\rho_{v w}^{2}\right)}\left[\frac{\left(r-\mu_{v}\right)^{2}}{\sigma_{v}^{2}}-2 \rho_{v w} \frac{\left(r-\mu_{v}\right)\left(s-\mu_{w}\right)}{2 \sigma_{v} \sigma_{w}}+\frac{\left(s-\mu_{w}\right)^{2}}{\sigma_{w}^{2}}\right]\right\} d r d s \\
C_{G}\left(F(w), F(u), \rho_{w u}\right)=\int_{-\infty}^{F^{-1}(w) F^{-1}(u)} \int_{-\infty} \frac{1}{2 \pi \sigma_{w} \sigma_{u} \sqrt{1-\rho_{w u}^{2}}} \\
\cdot \exp \left\{-\frac{1}{2\left(1-\rho_{w u}^{2}\right)}\left[\frac{\left(r-\mu_{w}\right)^{2}}{\sigma_{w}^{2}}-2 \rho_{w u} \frac{\left(r-\mu_{w}\right)\left(s-\mu_{u}\right)}{2 \sigma_{w} \sigma_{u}}+\frac{\left(s-\mu_{u}\right)^{2}}{\sigma_{u}^{2}}\right]\right\} d r d s
\end{array}\right.
$$

The density functions of Gaussian copula functions $C_{G}(F(u), F(v))$ can be written as $c(F(u), F(v))$; The density functions of GCF $C_{G}(F(v), F(w))$, can be written as $c(F(v), F(w))$, The density functions of GCF $C_{G}(F(w), F(u))$ can be written as $c(F(w), F(u))$ respectively and let $f(u), f(v), f(w)$ as the density functions of marginal distribution functions $F(u), F(v)$,

$F(w)$ respectively. So, according to statistics theory the density function of $\mathrm{F}(\mathrm{u}, \mathrm{v}), F(v, w), F(w, u)$ are expressed as followed:

$$
\left\{\begin{array}{l}
f\left(u, v, \rho_{u v}\right)=c(F(u), F(v)) \cdot f(u) \cdot f(v) \\
f\left(v, w, \rho_{v w}\right)=c(F(v), F(w)) \cdot f(v) \cdot f(w) \\
f\left(w, u, \rho_{w u}\right)=c(F(w), F(u)) \cdot f(w) \cdot f(u)
\end{array}\right.
$$

Where: 


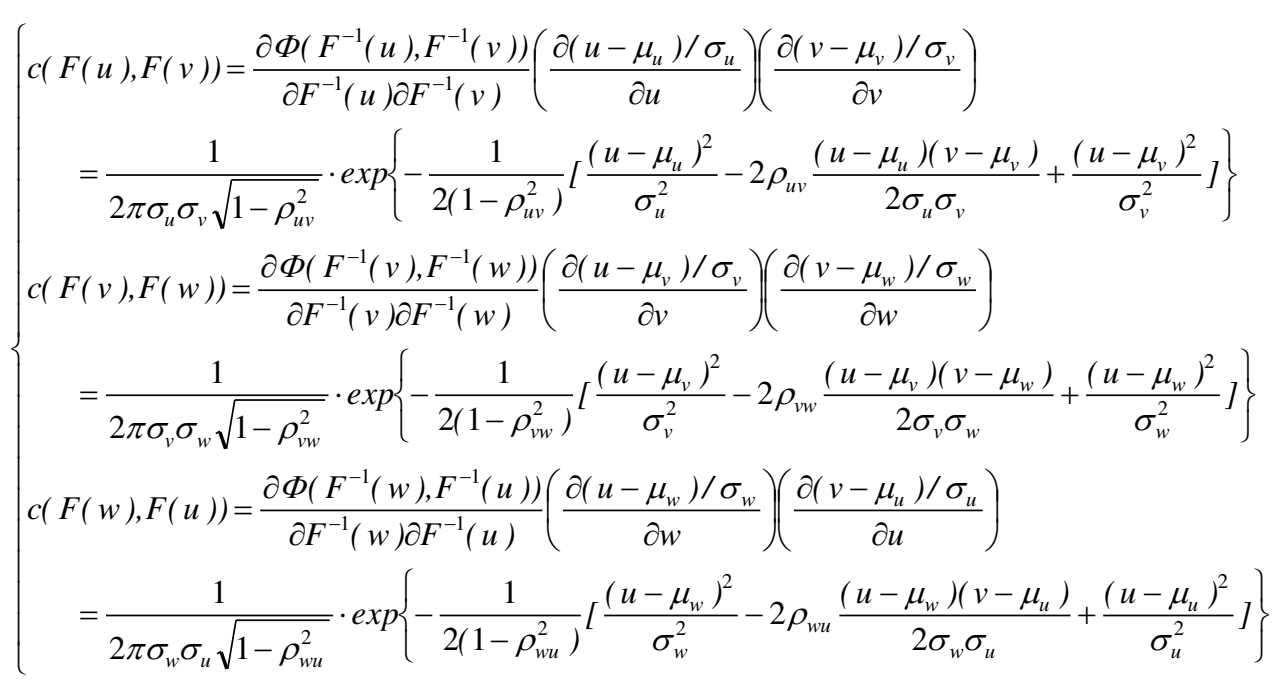

\subsection{Parameters estimating}

For the specified distribution of stochastic variable, the maximum likelihood method is a widely used parameter estimation method[19].

Therefore, The method of Maximum Likelihood can be introduced to estimate parameters of (15), (16). For easily resolving it, take the logarithms on both sides of the equation and log-likelihood function of the joint distribution function can be gotten as:

$$
\left\{\begin{array}{l}
\ln L\left(f\left(u, v, \rho_{u v}\right)\right)=\pi f\left(u, v, \rho_{u v}\right)=\sum_{n=1}^{N}\left[\ln f\left(u_{n}\right)+\ln f\left(v_{n}\right)\right]+\sum_{n=1}^{N} \ln c_{n}(F(u), F(v)) \\
\ln L\left(f\left(v, w, \rho_{v w}\right)\right)=\pi f\left(v, w, \rho_{v w}\right)=\sum_{n=1}^{N}\left[\ln f\left(v_{n}\right)+\ln f\left(w_{n}\right)\right]+\sum_{n=1}^{N} \ln c_{n}(F(v), F(w)) \\
\ln L\left(f\left(w, u, \rho_{w u}\right)\right)=\pi f\left(w, u, \rho_{w u}\right)=\sum_{n=1}^{N}\left[\ln f\left(w_{n}\right)+\ln f\left(u_{n}\right)\right]+\sum_{n=1}^{N} \ln c_{n}(F(w), F(u))
\end{array}\right.
$$

Substituting the above 150 groups data into the first formula, the parameter $\rho_{u v}$ can be calculated and the result was:

$$
\rho_{u v}=0.0798
$$

In the same way, Substituting the above 150 groups data into the second and the third formula,the parameters $\rho_{v w}, \rho_{w u}$ can be calculated

and the result were::

$$
\rho_{v w}=0.2615 ; \quad \rho_{w u}=0.3826
$$

Substitute Eq. (15) into Eq. (9), the density function can be spread as: 


$$
\left\{\begin{aligned}
f\left(y_{1}, \mu_{1}, \sigma_{1}\right) & =\lambda_{1} c(F(u), F(v)) \cdot f(u) \cdot f(v) \\
& +\lambda_{2} c(F(v), F(w)) \cdot f(v) \cdot f(w)+\lambda_{3} c(F(w), F(u)) \cdot f(w) \cdot f(u) \\
f\left(y_{2}, \mu_{2}, \sigma_{2}\right) & =\kappa_{1} c(F(u), F(v)) \cdot f(u) \cdot f(v) \\
& +\kappa_{2} c(F(v), F(w)) \cdot f(v) \cdot f(w)+\kappa_{3} c(F(w), F(u)) \cdot f(w) \cdot f(u)
\end{aligned}\right.
$$

From the section 3.2.1, The variables y1, y2 follow the normal distribution and their distribution function and density function can be

expressed as:

$$
\begin{aligned}
& \left\{\begin{array}{l}
F\left(y_{1}\right)=\Phi\left(\frac{\mu-\mu_{1}}{\sigma_{1}}\right) \\
F\left(y_{2}\right)=\Phi\left(\frac{\mu-\mu_{2}}{\sigma_{2}}\right)
\end{array}\right. \\
& \left\{\begin{array}{l}
f\left(y_{1}\right)=\varphi\left(\frac{u-u_{1}}{\sigma_{1}}\right) \\
f\left(y_{2}\right)=\varphi\left(\frac{u-u_{2}}{\sigma_{2}}\right)
\end{array}\right.
\end{aligned}
$$

the expression Eq. (20). can be spread as:

$$
\left\{\begin{array}{l}
f\left(y_{1}, \mu_{1}, \sigma_{1}\right)=\frac{1}{\sqrt{2 \pi} \sigma_{1}^{2}} \exp \left(\frac{\left(y_{1}-\mu_{1}\right)^{2}}{2 \sigma_{1}^{2}}\right) \\
f\left(y_{2}, \mu_{2}, \sigma_{2}\right)=\frac{1}{\sqrt{2 \pi} \sigma_{2}^{2}} \exp \left(\frac{\left(y_{2}-\mu_{2}\right)^{2}}{2 \sigma_{2}^{2}}\right)
\end{array}\right.
$$

Similarly, Eq. (6) can be spread as:

$$
\left\{\begin{array}{l}
f\left(u, \mu_{u}, \sigma_{u}\right)=\frac{1}{\sqrt{2 \pi} \sigma_{u}^{2}} \exp \left(\frac{\left(u-\mu_{u}\right)^{2}}{2 \sigma_{u}^{2}}\right) \\
f\left(v, \mu_{v}, \sigma_{v}\right)=\frac{1}{\sqrt{2 \pi} \sigma_{v}^{2}} \exp \left(\frac{\left(v-\mu_{v}\right)^{2}}{2 \sigma_{v}^{2}}\right) \\
f\left(w, \mu_{w}, \sigma_{w}\right)=\frac{1}{\sqrt{2 \pi} \sigma_{w}^{2}} \exp \left(\frac{\left(w-\mu_{w}\right)^{2}}{2 \sigma_{w}^{2}}\right)
\end{array}\right.
$$

Substitute Eq. (15), Eq. (16), Eq. (19), Eq. (20) into the first equation in Eq. (18) , then, the first equation of Eq. (18) was spread as followed: 


$$
\begin{aligned}
\frac{1}{\sqrt{2 \pi} \sigma_{1}^{2}} \exp \left(\frac{\left(y_{1}-\mu_{1}\right)^{2}}{2 \sigma_{1}^{2}}\right) & =\frac{1}{2 \pi \sigma_{u} \sigma_{v} \sqrt{1-\rho_{u v}}} \cdot \exp \left\{-\frac{1}{2\left(1-\rho_{u v}^{2}\right)}\left[\frac{\left(u-\mu_{u}\right)^{2}}{\sigma_{u}^{2}}-2 \rho_{u v} \frac{\left(u-\mu_{u}\right)\left(v-\mu_{v}\right)}{2 \sigma_{u} \sigma_{v}}+\frac{\left(v-\mu_{v}\right)^{2}}{\sigma_{v}^{2}}\right]\right\} \\
& +\frac{1}{2 \pi \sigma_{v} \sigma_{w} \sqrt{1-\rho_{v w}}} \cdot \exp \left\{-\frac{1}{2\left(1-\rho_{v w}^{2}\right)}\left[\frac{\left(v-\mu_{v}\right)^{2}}{\sigma_{v}^{2}}-2 \rho_{u v} \frac{\left(v-\mu_{v}\right)\left(w-\mu_{w}\right)}{2 \sigma_{v} \sigma_{w}}+\frac{\left(w-\mu_{w}\right)^{2}}{\sigma_{w}^{2}}\right]\right\} \\
& +\frac{1}{2 \pi \sigma_{w} \sigma_{u} \sqrt{1-\rho_{w u}}} \cdot \exp \left\{-\frac{1}{2\left(1-\rho_{w u}^{2}\right)}\left[\frac{\left(w-\mu_{w}\right)^{2}}{\sigma_{w}^{2}}-2 \rho_{w u} \frac{\left(w-\mu_{w}\right)\left(u-\mu_{u}\right)}{2 \sigma_{w} \sigma_{u}}+\frac{\left(u-\mu_{u}\right)^{2}}{\sigma_{u}^{2}}\right]\right\}
\end{aligned}
$$

Substituting the values of $\rho_{u v}, \rho_{v w}, \rho_{w u}$ and 150 groups data in the table 1 into Eq. (23), and then, Substituting Eq. (21), Eq. (22), Eq. (23) into Eq. (18). The least square method was applied to parameters calculating and then, the value of parameters $\lambda_{i}(i=1,2,3)$ can be gained as followed:

$$
\lambda_{1}=0.2836 ; \quad \lambda_{2}=0.0028 ; \quad \lambda_{3}=0.0097 .
$$

In like manner, the value of parameters $\kappa_{i}(i=1,2,3)$ of the second equation in Eq. (18) can be got as follows:

$\kappa_{1}=0.0086 ; \quad \kappa_{2}=0.0735 ; \quad \kappa_{3}=0.0418$

Substitute the values of $\lambda_{i}(i=1,2,3), \kappa_{i}(i=1,2,3)$ into Eq. (8) and the math modeling of the distribution of the radial run-out of the bearings and the ending beat can be obtained as follows:

$$
\begin{aligned}
F\left(y_{1}\right) & =0.2836 F(u, v) \\
- & 0.0028 F(v, w)+0.0097 F(w, u) \\
F\left(y_{2}\right) & =0.0086 F(u, v) \\
- & 0.0735 F(v, w)+0.0418 F(w, u)
\end{aligned}
$$

Substitute the values of $\lambda_{i}(i=1,2,3), \kappa_{i}(i=1,2,3)$ into Eq. (9) and the math modeling of Eq. (22) was gotten as followed:

$$
\begin{aligned}
& f\left(y_{1}, u_{1}, \sigma_{1}\right)=0.2836 f\left(u, v, \rho_{u v}\right) \\
& \quad-0.0028 f\left(v, w, \rho_{v w}\right)+0.0097 f\left(w, u, \rho_{w u}\right) \\
& f\left(y_{2}, u_{2}, \sigma_{2}\right)=0.0086 f\left(u, v, \rho_{u v}\right) \\
& \quad-0.0735 f\left(v, w, \rho_{v w}\right)+0.0418 f\left(w, u, \rho_{w u}\right)
\end{aligned}
$$

From the coefficient of the above mathematics modeling Eq. (24)and Eq. (25), the interaction with each other between parts of bearings can produce different effect on radial run out and ending beat of bearings. For the first equation of Eq. (24) or Eq. (25), the coefficient of the first item was the biggest one that was $0.2836(\max (0.2836,0.0028,0.0097))$, that meant the interaction with each other between the inner ring and outer ring can produce the greatest effect on radial run out, then, the effect between the rollers and inner ring, the last, the effect between the rollers and the outer ring. For the second equation of Eq. (24) or Eq. (25), the coefficient of the second item was the biggest one that was 0.0735 (max 
$(0.0086,0.0735,0.0418))$, that meant the interaction with each other between the inner ring and the rollers can produce the biggest effect on the the ending beat, then , the effect between the rollers and the outer ring, the last, the effect between the outer ring and the inner ring. This shows that when bearing parts are being processed, the upper deviation of outer ring and the lower deviation of inner ring need to be controlled strictly besides controlling dimension in standard tolerance, simultaneously, the rollers must also be strictly selected to guarantee their uniformity.

In general, it is after the parts are assembled into the bearings that can the radial run-out and the ending beat of bearings come into being. According to the above method, as long as the dimensions precision of inner ring, outer ring and rollers are known, the radial and end beat range of the bearing can be calculated before assembling. This meant once the parts precision is determined, the kinematic accuracy of bearing will be sure.

Of course, the above mathematics modeling were built according to the data from NU1004 bearings. For other type bearings, the distribution law of the dimension of parts may be different, the mathematics model may be also different. It need construct corresponding modeling from the relevant parts. But the copula method can be used for any bearings.

\section{Experimental Verifying}

To verify the above conclusion, we have done experiment with the same type bearings (NU1004 ). the other 150 groups parts of the bearings were taken randomly, numbered, measured respectively and the other 150 groups data of $\mathrm{u} 、 \mathrm{v} 、 \mathrm{w}$ were obtained by the number. Similarly, because of the limited space, only the 10 groups data were listed in table.4. Substituting this 150 groups data into the first equation of (25) and solving it and the mean values $\mu_{1}$ and the standard deviation $\sigma_{l}$ can be gotten. In the same way, Substituting this 150 groups data into the second equation of (25) and solving it and the mean values $\mu_{2}$ and the standard deviation $\sigma_{2}$ can be yielded. According to the parameters $\mu_{1} \mu_{2}$ and $\sigma_{1} \mu_{2}$, it can be known that the radial run out and the ending beat of bearings will be in a specific scope after assembling. To verify this result, the 150 groups parts were assembled according to their No. After the radial run out and the ending beat of bearings were measured, the mean values $\hat{\mu}$ and the standard deviation $\hat{\mu}$ can be evaluated by statistic software R. the computed value by (25) and the evaluated value were list in table.5. From table.5, the computed value by modeling (25) and the evaluated value by measured data is 
approximately unanimous. There was only difference from $0.0001 \mu \mathrm{m}$ to $0.0003 \mu \mathrm{m}$ between two results.

Table 4. ten groups data for verifying

\begin{tabular}{rrrrrr}
\hline No. & $y_{1}(\mu m)$ & $\mathrm{y}_{2}(\mu m)$ & $u(\mu m)$ & $v(\mu m)$ & $w(\mu m)$ \\
\hline 1 & 4.5 & 3.2 & 5.5 & 4 & 1.2 \\
2 & .1 .8 & 10.5 & 5.8 & 2.8 & 1.0 \\
3 & 8.0 & 9.5 & 7.5 & 6.0 & 1.4 \\
4 & 6.8 & 6.0 & 4.5 & 13.2 & 1.2 \\
5 & 11.2 & 8.5 & 15.2 & 8.5 & 2.6 \\
6 & 5.0 & 2.5 & 5.8 & 3.0 & 1.0 \\
7 & 15.3 & 15.0 & 12.0 & 8.3 & 2.6 \\
8 & 12.0 & 16.8 & 12.0 & 8.0 & 2.6 \\
9 & 2.1 & 14.5 & 6.5 & 0.0 & 0.5 \\
10 & 5.5 & 7.0 & 6.5 & 6.5 & 1.5 \\
\hline
\end{tabular}

Table 5 the comparing result of measuring and computing

\begin{tabular}{|c|c|c|c|c|}
\hline \multirow[t]{2}{*}{ Run-out } & \multicolumn{2}{|c|}{ measuring data $(\mu \mathrm{m})$} & \multicolumn{2}{|c|}{ computing result $(\mu \mathrm{m})$} \\
\hline & Mean value $\hat{\mu}$ & standard deviation $\hat{\sigma}$ & Mean value $\mu$ & standard deviation $\sigma$ \\
\hline$y_{1}$ & 7.8864 & 5.1478 & 7.8865 & 5.1478 \\
\hline$y_{2}$ & 6.1641 & 2.1621 & 6.1644 & 2.2621 \\
\hline
\end{tabular}

\section{Conclusions}

(1) the coordination among the parts of bearings is very important. The kinematic accuracy of bearings mainly come from the interaction with multiple parts. In general, it is often considered that the high grade precision bearings consist of high precision parts. But that is not the case, the harmonization among its parts is more important.

(2) From the mathematics modeling in the paper, for the radial run out, the harmonization between the inner ring and the outer ring is very important, while for the ending beat, the harmonization between the rollers uniformity and the inner ring should be paid more attention. Therefore, when the radial beat accuracy of bearings must be requested, the harmonization between the inner ring and the outer ring have to be controlled strictly. When the ending beat accuracy of bearings must be requested, the harmonization between the rollers uniformity and the inner ring need to be controlled strictly.

(3) Bearing is a system consisted of multiple components. The radial run out and the ending beat are main indices of the kinematic accuracy. 
The interact with each other among parts exerts joint influence on the radial run-out and the ending beat of bearings and then on kinematic accuracy. The method of distribution estimation algorithm with CJDF was be introduced to constructing the math modeling between the precision of parts and the radial and ending beat of bearing. With this modeling, the kinematic accuracy of bearing can be forecast by the actual dimension deviation of parts.

\section{Declarations}

\section{Availability of data and material}

The method in this paper is applicable for all type bearings, but the data and materials in this paper are only applicable for the bearing which type is UN1004. For other type bearings, that need corresponding data.

\section{Conflict of interest}

There are no any competing interests to others

\section{Funding}

This work is supported by the National Key R\&D Program of China (No. 2019YFB2005004) which was received by Xue Yujun. The measuring data over UN1004 were kindly provided by Xue Yujun.

\section{Authors' contributions}

Lihong Li, primary author, she have mainly finished analyzing and writing paper. Yujun Xue , he join in the topic discussion and collect material and measuring data. Jishun LI, he had mainly proposed guidance and a lot of advice. All authors read and approved the final manuscript.

\section{Acknowledgements}

The first author would like to thank Professor Xue Yujun and Professor LI Jishun for their advice on this research and will gratefully acknowledge the comments and suggestions of all reviewers.

\section{Authors' information}

Lihong Li, born in 1965, is a associate professor at Vehicle \& Transportation Engineering institute, Henan University of Science and 
Technology,China. Her research interests include mechine engineering and transportation energy.

Tel: +8615896539872

E-mail:lyh_5204@163.com

Yujun Xue, born in 1971, is currently a professor at Henan Key Lab for Machinery Designed Transmission System, Henan University of

Science and Technology, China. His research interests include man-machine system and intelligent robotics.

E-mail: xue_yujun@163.com

Jishun LI, born in 1963, is currently a professor at Henan Key Lab for Machinery Designed Transmission System, Henan University of

Science and Technology, China. His research interests include man-machine system and intelligent robotics

E-mail: li_jishun@163.com

\section{Reference}

[1] Rudnicki, Mariusz; Marszal, Jacek; Salamon, Roman.(2020). Impact of Spatial Noise Correlation on Bearing Accuracy in DIFAR Systems[J]. Archives of Acoustics, 45(4): p 709-720,.

[2] NiDong Yang, Shiwen; Chen, Yikai; Yang, Feng.(2017). Direction finding based on TMAs with reconfigurable angle-searching range and bearing accuracy. Electronics Letters, 53( 3): 130-132.

[3] Minhas, Amrinder Singh,etc.(2020)..Improvement in classification accuracy and computational speed in bearing fault diagnosis using multiscale fuzzy entropy. Journal of the Brazilian Society of Mechanical Sciences and Engineering,42(11): 623-635

[4] Zha, Jun,Chen, Yaolong.(2020). Effect of design parameters and operational conditions on the motion accuracy of hydrostatic thrust bearing[J]. Proceedings of the Institution of Mechanical Engineers, Part C: Journal of Mechanical Engineering Science, 234( 8): 1481-1491

[5] Zha, Jun Chen, Yaolong and etc.( 2017). Relationship between elliptical form error and rotation accuracy of hydrostatic journal bearing. Industrial Lubrication and Tribology, 69( 6): 905-911.

[6] CHEN G C, WANG B K , MAO F H.( 2013). Effects of raceway roundness and roller diameter errors on clearance and runout of a cylindrical roller bearing[J]. Proceedings of the Institution of Mechanical Engineers. Part J: Journal of Engineering Tribology.

[7] SHOJI N, Tohru Kanada.(2018). Development of measuring system for radial non-repetitive run-out (NRRO) and perception about present state of angular contact ball bearing for machine tools . Tribology International, 41(12):1176 -1180.

[8] OKAMOTO, J. (2001). Study on run-out of ball bearings-relation between unroundness of race and locus of shaft in rotation. Journal of Japanese Society of Tribologists, 46(7):578-584.

[9] Shi Wenxiang, Li Jishun, Liu Yonggang.(2012). Forecast and Simulation of Rotational Accuracy of Cylindrical Roller Bearing . Mechanical Science 
and Technology for Aerospace Engineering, 31(4) : 612-616.

[10] YANGZhao-hui, ZHANGJin-hua, HongJun etc.(2014). Errors analysis of tra-precision measurement system Forratational accuracy of rolling bearings. Journal of Zhejing University (Engineering Science), 48(6):1095-1101

[11] Shi Wenxiang,2011. Calculation and statistical analysis of bearing running accuracy based on contour shape of outer ring. MS thesis, Henan: Henan university of science and technology, China.

[12] Songfei. (2012). The prediction of bearing rotative accuracy base on inner ring profile and radial load. MS thesis, Henan: Henan university of science and technology ,China.

[13] NOGUCHI J, HIRUMA K, KAWA H. (2005).The influence of location of balls and ball diameter difference in rolling bearings on the nonrepetitive runout (NRRO) of retainer revolution. Tribology International, 29(1):11-18.

[14] WU Quan-you,2015. Ananlysis of cylindrical roller bearing accuracy under the influence of bearing parts dimension error. MS thesis, Henan: Henan university of science and technology, China.

[15] Li-hong Li, Ji-shun Li, Yu-jun Xue and etc.(2017). "Distribution estimation method of the radial run-out of the antifriction bearing", 2017 International Conference on Advanced Mechatronic Systems (ICAMechS). Xiamen, China.

[16] R B.An Introduction to Copulas(Second Edition). New York:Springer-Verlag,2006 .

[17] CUI Yuwei, YUAN Pengcheng, NI Anning, JUAN Zhicai. (2014).Travel time reliability estimating methodology in transportation networks based on Copula function. Application Research of Computers, 31(5):1385-1389.

[18] Xiaolei Ma, Sen Luan, Bowen Du, Bin Yu. (2017). Spatial Copula Model for Imputing Traffic Flow Data from Remote Microwave Sensors. Sensors, $17(10), 2160$.

[19] ZHANG Qi-xin,WANG Shixing.( 2012). Application of the EDA Algorithm in the Near Range Optimal Rendezvous for Spacecrafts. COMPUTER ENGINEERING\&SCIENCE, 34(5):89-94.

[20] WU Hong,WANG Weiping,WANG Lei,YANG Fen.(2010). Application of EDA in Path Planning of Cruise Missile. Electronics optics \& control,17(7):6-10.

[21] LIN Jingli,YANGWeiming, YAN Gongjun.(2012). Cooperative collision warning based on Copula method. Application Research of Computers, 29(10):3761-3764.

[22] TANG Jiayin, HE Ping, LIANG Hongqin, WANG Qin.(2013). Comprehensive Reliability Assessment of Long-life Products with Correlated Multiple Failure Modes. Journal of mechanical engineering, 49(12):176-182.

[23] HE Chengming, WU Wei,MENG Qingjun.(2012). A Copula-based Mechanical System Reliability Model and Its Application. ACTA ARMAMENTARII, 33(3) : 379-384.

[24] XU A.(2012. Statistical Analysis of Competing Failure Modesin Accelerated Life Testing Based on Assumed Copulas. Chinese Journal of Applied Probability and Statistics, 28(6): 51-62.

[25] Dian-Qing Li, Lei Zhang, Xiao-Song Tang, Wei Zhou, Jin-Hui Li, Chuang-Bing Zhou. (2015). Bivariate distribution of shear strength parameters using copulas and its impact on geotechnical system reliability. Computers and Geotechnics 68: 184-195.

[26] Lu Wang. ( 2013). A high-dimensional vine copula approach to movement of China's financial markets. 2013 20th International Conference on 
Management Science and Engineering, 2013 : 1538-154.

[27] Selma Jayech. (2016). The contagion channels of July-August-2011 stock market crash:A DAG-copula based approach. European Journal of Operational Research 249: 631-646.

[28] JOSHUA V R, TIl S. A General Approach to Integrated risk management with Skewed, Fat-tailed Risks. Journal of Financial Economics,2006, 79(3): 569-614.

[29] Shui-Long Shen, Yong-Xia Wu, Ye-Shuang Xu, Takenori Hino.Huai-Na Wu. A Evaluation of hydraulic parameters from pumping tests in multi-aquifers with vertical leakage in Tianjin. Computers and Geotechnics. 2015, 68196-207

[30] TU Zhibin,HUANG Mingfeng,LOU Wenjuan. Dynamic wind load combination of tall buildings based on Copula functions[J]. Journal of Zhejiang University (engineering science), 2014, 48(8):1370-1375.

[31] XU Yuqin,ZHANG Linban. Modeling of wind speed correlation and analysis of probabilistically optimal power flow based on Copula function. Journal of Noah China Electric Power University, 2013, 40(5):54-59 .

[32] Alireza Ahmadabadi, Burcu HudaverdiUcer. (2017). Bivariate nonparametric estimation of the Pickands dependence function using Bernstein copula with kernel regression approach. Comput Stat, 32:1515-153

[33] Li Qiang. (2012).The Study of Financial Market Risk Measurement Based on Copula theory and GPD Model[D], Chongqing University, China.

[34] Zhong bo, Sun yoingbo.(2011,). On the effect of copula for reliability of parallel system with dependent components. Journal of applied statistics and management, 30(2): 363-369

[35] Gildas M, Stéphane G, Florence F. (2015). A Class of Multivariate Copulas Based on Products of Bivariate Copulas. Journal of Multivariate Analysis, 140(8):363-376.

[36] Nob Y , Choi K K , (2009). Du Liu . Reliability-Based Design Optimization of Problems with Correlated Input Variables Using a Gaussian Copula . Struet Multidisc Optim, 38(6 ) :1-16.

[37] YU Yongjian LI Jishun CHEN Guoding and etc. (2016). Numerical Calculation and Experimental Research of Rotational Accuracy on Cylindrical Roller Bearing. Bearing, JOURNAL OF MECHANICAL ENGINEERING, 52(15):65-71

[38] Marcelo Brutti Righi, Paulo Sergio Ceretta. (2015) . Forecasting Value at Risk and Expected Shortfall based on serial pair-copula constructions. Expert Systems with Applications , $42: 6380-6390$

[39] Shouxiang Wang, Xingyou Zhang, Liyang Liu. (2016). Multiple stochastic correlations modeling for microgrid reliability and economic evaluation using pair-copula function. Electrical Power and Energy Systems, 76: 44-52

[40] Luciana Dalla Valle, Maria Elena De Giuli, Claudia Tarantola, Claudio Manelli. (2016). Default probability estimation via pair copula constructions. European Journal of Operational Research, 249: 298-311 